\title{
IMAGE PREPROCESSING FOR CBIR SYSTEM
}

\author{
Tatiana Jaworska \\ Systems Research Institute Polish Academy of Sciences, Newelska 6 St, 01-447 Warsaw, Poland \\ Tatiana.Jaworska@ibspan.waw.pl
}

\begin{abstract}
Keywords: Content-based image retrieval (CBIR), image preprocessing, image segmentation, clustering, object extraction, texture extraction, discrete wavelet transformation.

Abstract: $\quad$ This article describes the way in which image is prepared for content-based image retrieval system. Our CBIR system is dedicated to support estate agents. In our database there are images of houses and bungalows. All efforts have been put into extracting elements from an image and finding their characteristic features in the unsupervised way. Hence, the paper presents segmentation algorithm based on a pixel colour in RGB colour space. Next, it presents the method of object extraction in order to obtain separate objects prepared for the process of introducing them into database and further recognition. Moreover, a novel method of texture identification which is based on wavelet transformation, is applied.
\end{abstract}

\section{INTRODUCTION}

Image processing for purposes of content-based image retrieval (CBIR) systems seems to be a very challenging task for the computer. Determining how to store images in big databases, and later, how to retrieve information from them, is an active area of research for many computer science fields, including graphics, image processing, information retrieval and databases.

Although attempts have been made to perform CBIR in an efficient way based on shape, colour, texture and spatial relations, it has yet to attain maturity. A major problem in this area is computer perception. There remains a big gap between lowlevel features like shape, colour, texture and spatial relations, and high-level features like windows, roofs, flowers, etc.

The purpose of this paper is to investigate image processing with special attention given to segmentation and selection of separate objects from the whole image. In order to achieve this aim we present two new methods: one is a very fast algorithm for colour image segmentation, and the second is a new approach to description of textured objects, using discrete wavelet transformation.

\section{CBIR CONCEPTION OVERVIEW}

In the last 15 years, CBIR techniques have drawn much interest, and image retrieval techniques have been proposed in context of searching information from image databases. In the 90's the Chabot project at UC Berkeley (Ogle, 1995) was initialized to study storage and retrieval of a vast collection of digitized images. Also, at IBM Almaden Research Centre CBIR was prepared by Flickner (Flickner, 1995), Niblack (Niblack, 1993). This approach was improved by Tan (Tan, 2001), Hsu (Hsu, 2000) and by Mokhtarian, F. S. Abbasi and J. Kittler (Mokhtarian, 1996) at Department of Electronics and Electrical Engineering UK.

Our CBIR system is dedicated to support estate agents. In the estate database there are images of houses, bungalows, and other buildings. To be effective in terms of presentation and choice of houses, the system has to be able to find the image of a house with defined architectural elements, for example: windows, roofs, doors, etc. (Jaworska, 2005).

The first stage of our analysis is to split the original image into several meaningful clusters; each of them provides certain semantics in terms of human understanding of image content. Then, proper features are extracted from these clusters to represent the image content on the visual perception level. In the interest of the following processes, such 
as object recognition, the image features should be selected carefully. Nevertheless, our efforts have been put into extracting elements from an image in the unsupervised way.

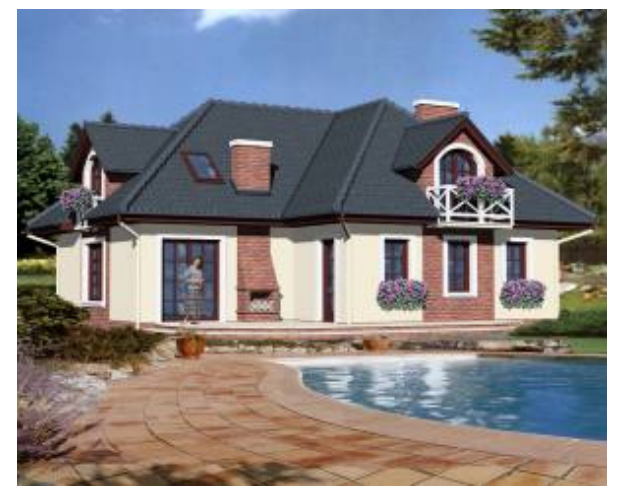

Figure 1: Example of an original image.

\section{A NEW FAST ALGORITHM FOR OBJECT EXTRACTION FROM COLOUR IMAGES}

We definitely prefer unsupervised techniques of image processing. Although there are many different methods of image segmentation, we began with two well known clustering algorithms: the C-means clustering (Seber,1984), (Spath, 1985), and later developed, the fuzzy C-means clustering algorithm (FCM) (Bezdek, 1981). In our case we found clusters in the 3D colour space RGB and HSV.

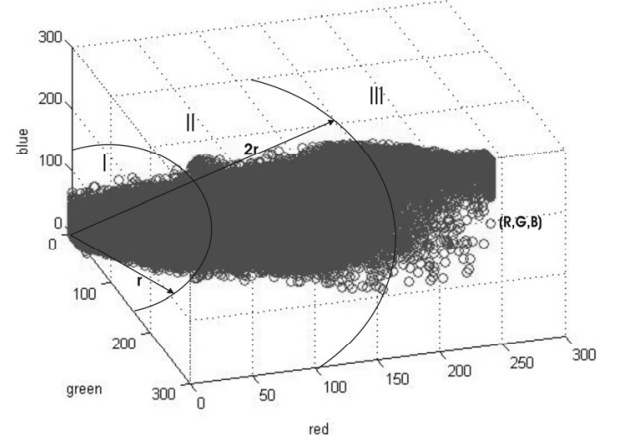

Figure 2: The way of labelling the set of pixels. Regions I, II, III show pixel brightness and the biggest value of triple $(\mathrm{R}, \mathrm{G}, \mathrm{B})$ determines its colour.

Unfortunately, results were unsatisfying. After examining the point distribution in these both spaces (for all images) it turned out that points created one tight set. In figure 2 such a set is exemplified in
RGB space but points distribution in HSV space is similar.

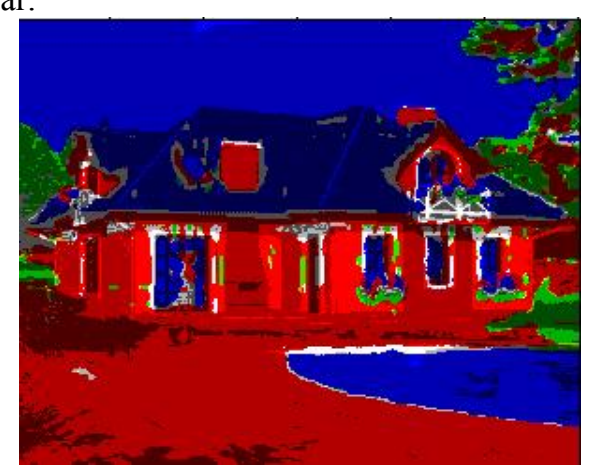

Figure 3: 12 cluster segmentation of fig. 1 obtained by using the 'colour' algorithm.

These results forced us to work out a new algorithm which uses colour information about a single point to greater extent than the C-means algorithm does. With the aim of labelling a pixel we chose the biggest value from the triple $(\mathrm{R}, \mathrm{G}, \mathrm{B})$ and we defined it as a cluster colour. In this way we obtained three segments - red, green and blue and for better result we divided each colour into three shades, according to the darkness of colour shown as three regions (I, II, III) which determine point brightness. The idea of the segmentation is illustrated in figure 2. The radius $r=\sqrt{R_{\max }^{2}+G_{\max }^{2}+B_{\max }^{2}} / 3$ of the dividing sphere was counted in Euclidean measure, where $R_{\max }=G_{\max }=B_{\max } \# 255$. Moreover, we added three segments: black, grey and white for pixels for whom $\mathrm{R}=\mathrm{G}=\mathrm{B}$ according to their region (I, II, III). We called this algorithm 'colour one'.

Figure 3 presents the image shown in fig. 1 divided into 12 clusters using the above-described algorithm.

\section{OBJECT EXTRACTION ON THE BASE OF THE NEW ALGORITHM}

Based on this segmentation separate objects are obtained. As an object we understand an image of architectural element such as roof, chimney, door, window, etc.

After performing the extraction of objects, the following features for these objects were counted: average colour (shown in fig. 4), texture parameters, region-based shape descriptors, contour based shape descriptors and location in the image as a regionbased representation. 


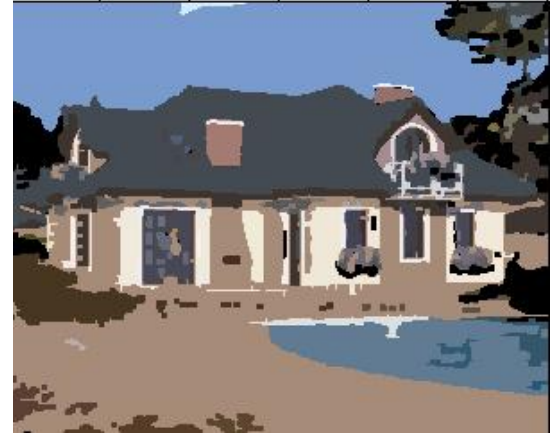

Figure 4: Objects from fig. 2 presented in their average colours.

\section{THE DETERMINATION OF TEXTURE PARAMETERS}

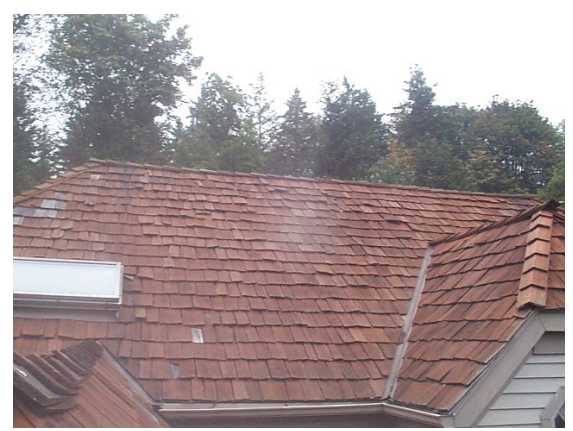

Figure 5: Example of an original image where the roof is a textured surface.

The texture information presented in images is one of the most powerful additional tools available. There are many methods which can be used for texture characterization. Unfortunately, they are mostly useless for our purpose.

One of them is the two-dimensional frequency transformation. For our aims we could apply as well the classical Fourier transformation as several spatial-domain texture-sensitive operators, for instance, the Laplacian $3 \times 3$ or $5 \times 5$, the Gaussian $5 \times 5$, Hurst, Haralick, or Frei and Chen (Russ, 1995). Regrettably, all of them are useful for relatively small neighbourhoods.

The other method of texture recognition for monochromatic image is the histogram thresholding. Unfortunately, it can be used mainly for distinguishing 2-3 textured regions. There also exists the twodimensional histogram of pixel pairs proposed by Haralick in 1973 (Haralick, 1973).

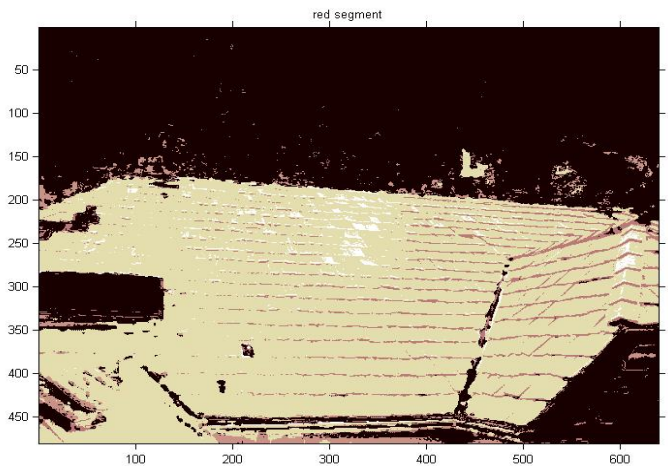

Figure 6: The red segment (in three levels of brightness) extracted from the whole segmentation from fig. 8 .

The next methods are the transformation domain approaches. In 2001 Balmelli and Mojsilović (Balmelli, 2001) proposed the wavelet domain for texture and pattern using statistical features only for regular textures and geometrical patterns. So far only Lewis and Fauzi manage to perform an automatic texture segmentation for CBIR based on discrete WT (DWT) (Fauzi, 2006).

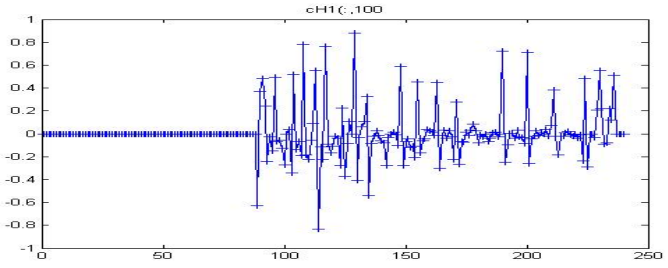

Figure 7: Horizontal wavelet coefficients presented along the $100^{\text {th }}$ column of the image transform (for the Haar wavelet, where $j=1$ ). Numbers of the Haar wavelets for the first level of multiresolution analysis are on the horizontal axis and values of coefficients $\mathrm{cH} 1$ are on vertical axis.

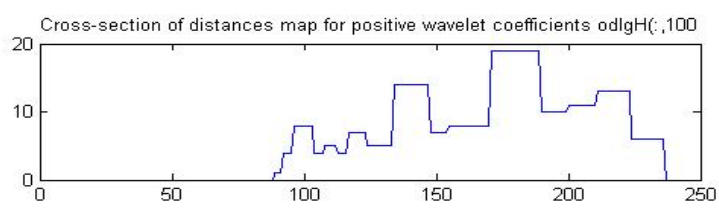

Figure 8: Cross-section through the $100^{\text {th }}$ column of the distances map for positive horizontal wavelet coefficients. Numbers of the Haar wavelets for the first level of multiresolution analysis are on the horizontal axis and distances between the maximal wavelet coefficients are on vertical axis.

In our work we decided to use the Fast Wavelet Transform (FWT). It is efficient and productive enough for frequent use for our purpose.

One of the most important features of details is their directionality. If we use this feature and compute the convolution of an image consisting of 
regular tiles or bricks and relevant wavelet, we obtain a $2 \mathrm{D}$ transform whose maximum values are placed in the connection spots among these tiles or bricks.

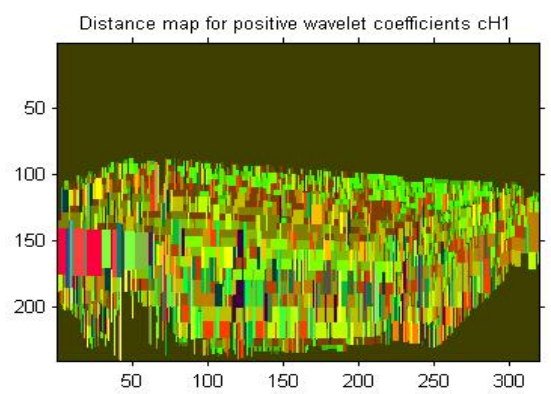

Figure 9: Distance map for positive horizontal wavelet coefficients $\mathrm{cH} 1$. There are wavelet numbers on both axes.

Therefore, we have applied the Haar wavelet to the roof region shown in fig. 7. Then, we obtained three matrices of details $d_{1}^{1}, d_{1}^{2}$ and $d_{1}^{3}$. The cross-section through the $100^{\text {th }}$ column of the horizontal details matrix $d_{1}^{1}(\mathrm{cH} 1)$ is presented in figure 8. Maxima and minima in this figure are equivalent to connections between tiles in fig. 7. Having computed horizontal details, we have measured distances between maxima for each column of this matrix (shown in fig. 8) and we have measured distances between minima for each column of this matrix. We have located one threshold on the level of $1 \%$ of the maximum value of the whole matrix and we have measured distances between positive coefficients on that level and we have done analogically for negative coefficients. It has turned out that these distances which are equivalent to the size of tiles are good distinctive parameters for textured region.

After counting the distances we have created two distance maps for all positive and negative horizontal coefficients. Figure 9 presents one of these distance maps. Analogical procedure has been carried out for vertical wavelet coefficients $\mathrm{cV} 1$. Basing on the above distance maps we can estimate that the size of tiles.

\section{CONCLUSIONS}

To sum up, this paper shows how to extract elements from images in the unsupervised way and analyze objects parameters. We have focused on the description of texture parameters because it was the most difficult task. The achieved results indicate that it is possible to separate objects in the image with acceptable accuracy for further interpretation in the unsupervised way. In computer terms, objects are recognized by finding the above-mentioned features of each object and a new object is classified to one of the previous created classes. So far, we have no interpretation which of these objects are doors, windows, etc. At present, the database structure is being prepared. This structure will cover all elements necessary for image content analysis; namely basic object features as well as logical and spatial relations.

\section{REFERENCES}

Bezdek, J. C., 1981. Pattern Recognition with Fuzzy Objective Function Algorithms, Plenum Press, New York.

Fauzi, M., Lewis, P., 2006. Automatic texture segmentation for content-based image retrieval application, Pattern Analysis and Applications, Springer-Verlag, London, (in printing).

Flickner, M., Sawhney, H., et al., 1995. Query by Image and Video Content: The QBIC System, IEEE Computer, Vol. 28, No. 9, pp. 23-32.

Haralick, R. M., Shanmugan, K., Dinstein, I., 1973. Texture Features for Image Classification, IEEE Transactions of Systems, Man and Cyberntics, SMC-3, pp. 610-621.

Hsu, W., Chua, T. S., Pung, H. K., 2000. Approximation Content-based Object-Level Image Retrieval, Multimedia Tools and Applications, Vol. 12, Springer Netherlands, pp. 59-79.

Jaworska, T., Partyka, A., 2005. Research: Content-based image retrieval system [in Polish], Report RB/37/2005, Systems Research Institute, PAS.

Mokhtarian, F., Abbasi, S., Kittler J., 1996. Robust and Efficient Shape Indexing through Curvature Scale Space, Proc. British Machine Vision Conference, pp. 53-62.

Niblack, W., Flickner, M., et al., 1993. The QBIC Project: Querying Images by Content Using Colour, Texture and Shape, SPIE, Vol. 1908, pp. 173-187.

Ogle, V., Stonebraker, M., 1995. CHABOT: Retrieval from a Relational Database of Images, IEEE Computer, Vol. 28, No 9, pp. 40-48.

Russ, J. C., 1995 The image processing. Handbook, $C R C$, London, pp. 361-385.

Seber, G., 1984. Multivariate Observations, Wiley.

Spath, H., 1985. Cluster Dissection and Analysis: Theory, FORTRAN Programs, Examples, translated by J. Goldschmidt, Halsted Press, pp. 226.

Tan, K-L., Ooi, B. Ch., Yee, Ch. Y., 2001. An Evaluation of Color-Spatial Retrieval Techniques for Large Image Databases, Multimedia Tools and Applications, Vol. 14, Springer Netherlands, pp. 55-78. 